\title{
Suivi sérologique de la vaccination contre les principales viroses aviaires dans les élevages de reproducteurs en Tunisie
}

\author{
A. Cherif ${ }^{1}$ A. Bouslama ${ }^{2}$ C. Chakroun ${ }^{2}$ I. Turki ${ }^{3 *}$ \\ K. Kaboudi ${ }^{3}$ M. Bouzouaia ${ }^{3}$
}

Mots-clés

Volaille - Poulet de chair - Animal reproducteur - Maladie de Newcastle - Maladie de Gumboro Bronchite infectieuse aviaire Vaccination - Elisa - Tunisie.

\begin{abstract}
Résumé
Une étude séro-épidémiologique a été menée au sein de six sociétés d'élevage de reproducteurs chair et quatre sociétés de reproducteurs ponte, couvrant la majeure partie des élevages aviaires reproducteurs en Tunisie. L'objectif a été d'évaluer l'efficacité de la réponse immunitaire suite à l'application des protocoles vaccinaux établis par les propriétaires et effectués contre les virus de la maladie de Newcastle (NDV), de la bronchite infectieuse (IBV) et de la maladie de Gumboro (IBD). Au cours des huit visites réalisées par élevage, des questionnaires ont été remplis et 2000 prélèvements ont été effectués. Ces échantillons ont été soumis au test Elisa indirect en utilisant les kits Civtest AVI-NDV, -IBV et -IBD. Pour chaque date et chaque maladie, les titres moyens en anticorps spécifiques ainsi que le coefficient de variation ont été calculés. Ces indicateurs ont permis de constater que le poussin était de bonne qualité sanitaire, la cinétique des anticorps postvaccinaux généralement satisfaisante et la réponse immunitaire globale suffisamment homogène. Toutefois, le protocole vaccinal recommandé par la Commission nationale de pathologie aviaire n'était pas toujours respecté, ce qui a entraîné soit une chute passagère des anticorps avec pour conséquence un risque élevé d'infection, soit des interventions supplémentaires aboutissant à des pertes économiques liées aux charges vaccinales. Ce travail montre aux éleveurs la nécessité de disposer d'une base de données pour la mise en place d'un programme de prophylaxie médicale spécifique aux conditions épidémiologiques de ces élevages.
\end{abstract}

\section{INTRODUCTION}

La méconnaissance du statut immunitaire des reproducteurs visà-vis de quelques infections virales rend difficile l'élaboration du programme de vaccination. Les éleveurs choisissent les programmes de vaccination en général sur la base de dates proposées par les fabricants de vaccins et éventuellement selon les recommandations

\footnotetext{
1. Institut de la recherche vétérinaire de Tunisie, Tunis, Tunisie.

2. Groupement inter-professionnel des produits avicoles et de la cuniculture, Tunis, Tunisie.

3. Ecole nationale de médecine vétérinaire, direction des Sciences cliniques, service des Maladies contagieuses, Sidi Thabet 2020, Tunisie.

* Auteur pour la correspondance

Tél. : +21671552 200 : fax : +21671552441

E-mail : turkiimed58@yahoo.fr
}

de la Commission nationale de pathologie aviaire (CNPA). En effet, la situation sanitaire en Tunisie est maitrisée grâce à l'implication de la commission et la collaboration de toutes les institutions, des services vétérinaires et des professionnels, appuyée par l'application d'une législation en la matière, ainsi que par le contrôle des maladies réputées contagieuses et les mesures de luttes contre la dissémination de celles-ci.

L'enquête sérologique menée dans les élevages de reproducteurs a eu deux objectifs. Le premier a consisté à évaluer le niveau global de la réponse immunitaire suite à la vaccination effectuée contre les virus de la maladie de Newcastle (NDV), de la bronchite infectieuse (IBV) et de la maladie de Gumboro (IBD), par un suivi de la cinétique des anticorps spécifiques révélés par la technique Elisa indirecte. Le second a consisté à calculer le coefficient de variation, pour apprécier l'homogénéité des réponses immunitaires induites, révélateur de problèmes d'application des techniques de vaccination utilisées. 


\section{MATERIEL ET METHODES}

\section{Visites des élevages et questionnaire}

Quatre sociétés de reproducteurs ponte et six de reproducteurs chair ont fait l'objet de cette enquête sérologique. Ces sociétés couvrent respectivement 100 et 56 p. 100 de la production nationale de poussins d'un jour ponte et chair (les 44 p. 100 restants représentent une multitude de petites sociétés). L'ensemble des sociétés retenues dans l'enquête pratiquaient l'élevage au sol, avec caillebotis, tout en respectant le système de la bande unique. Des mesures de prophylaxie sanitaire étaient mises en place par les différents centres visités. L'entrée de chaque exploitation était équipée d'un rotoluve contenant un désinfectant, tels que l'ammonium quaternaire ou l'eau de Javel régulièrement renouvelés. De même, des pédiluves étaient installés à l'entrée de chaque bâtiment. Les fenêtres des différents poulaillers étaient munies d'un grillage interdisant l'accès aux oiseaux sauvages et aux rongeurs, contre lesquels chaque société prévoyait un programme de dératisation.

Pour chaque bâtiment, huit visites ont été programmées. Le choix des dates de visite a tenu compte de nombreux paramètres tels que l'âge des oiseaux, les différentes vaccinations, le programme de vaccination préconisé, l'âge de l'entrée en ponte et la durée d'exploitation des animaux. Au vu de ces critères, les âges suivants ont été retenus : 1 jour et 7, 16, 22, 28, 44, 55, 68 semaines.

Des fiches d'enregistrement ont été élaborées, une fiche de visite d'élevage remplie à chaque intervention (figure 1), une fiche de prélèvements, et un tableau de relevés des vaccinations rempli par les propriétaires. Le questionnaire a été établi et axé sur la situation sanitaire et la prophylaxie médicale préalablement instaurée : type de vaccin (vivant ou inactivé), date et âge lors de la vaccination, méthode d'administration du vaccin.

\section{Fiche de visite - Reproducteur ponte}

Code

Etablissement

Adresse

Effectif de départ
$\mathrm{N}^{\circ}$ Laboratoire.

\section{Centre}

Date....

\begin{tabular}{|c|c|c|c|c|}
\hline \multirow{3}{*}{ VISITE } & \multirow{3}{*}{$\begin{array}{l}1 J_{-} \\
44 S_{-} \\
55 S_{-}\end{array}$} & \multirow{5}{*}{ SERUMS } & Bâtiment ... & Nb. sérums ...... \\
\hline & & & Bâtiment ... & Nb. sérums ...... \\
\hline & & & Bâtiment ... & Nb. sérums ....... \\
\hline \multirow{2}{*}{ ÂGE } & & & Bâtiment ... & Nb. sérums ...... \\
\hline & & & Bâtiment ... & Nb. sérums ....... \\
\hline
\end{tabular}

Vaccins conservés au centre : oui_ non___ Réfrigérateur : oui_ non_

Date de vaccination et vaccins

\begin{tabular}{|c|c|c|c|}
\hline Date & Vaccin et $n^{\circ}$ du lot & Mode de vaccination & Vaccinateur \\
\hline & & & \\
\hline & & & \\
\hline & & & \\
\hline & & & \\
\hline & & & \\
\hline & & & \\
\hline
\end{tabular}

Mortalité

Morbidité

Antécédents pathologiques

Traitements effectués

Manipulations, transferts

Problèmes sanitaires

Contrôle officiel : Date

Résultats : SPG

$\mathrm{MG}$

$\mathrm{MS}$

Auto-contrôle: Date

Résultats : SPG

$M G$

$\mathrm{MS}$

Autres

Observations :

Fait par :

Figure $\mathbf{1}$ : fiche de visite - reproducteur ponte. 


\section{Sérologie}

Les prélèvements ont été effectués au niveau de la veine alaire des volailles et réalisés directement dans l'élevage, à l'exception de ceux issus des poussins importés à l'âge d'un jour. Cette dernière catégorie de prélèvements a été faite au laboratoire par les responsables du contrôle officiel aux frontières.

A chaque visite, 20 à 30 prélèvements de sang ont été réalisés par bâtiment. Au total plus de 2000 échantillons ont été soumis aux analyses sérologiques. Ils ont été directement acheminés au laboratoire où ils ont subi le jour même une centrifugation en vue de récolter les sérums qui ont été par la suite conservés dans des microtubes, identifiés et congelés à $-18^{\circ} \mathrm{C}$.

La technique Elisa indirecte a été effectuée en utilisant des kits de la société LSI : Civtest AVI-NDV (NDV : virus de la maladie de Newcastle), Civtest AVI-IBV (IBV : virus de la bronchite infectieuse aviaire), Civtest AVI-IBD (IBD : virus de la maladie de Gumboro).

Les groupes de prélèvements effectués à différentes dates et provenant d'un même bâtiment ont été simultanément analysés avec le même kit afin d'assurer la comparabilité des résultats fournis par le test et de bien interpréter la cinétique des anticorps (Ac); les sérums ont été dilués au $1 / 500^{\mathrm{e}}$.

La lecture des plaques Elisa a été faite à l'aide d'un spectrophotomètre avec un filtre de $405 \mathrm{~nm}$. La densité optique (DO) obtenue a été transformée en titre d'Ac, la transformation des DO a été automatiquement calculée à l'aide d'un logiciel fourni par le laboratoire Hipra.

Le titre moyen de chaque lot de sérum a été calculé pour un élevage donné et pour une date déterminée. De même, l'évolution des titres a été illustrée par des graphes permettant de juger de l'efficacité des vaccins utilisés ainsi que de leur mode d'administration.

Pour pouvoir estimer le niveau d'hétérogénéité des lots, le coefficient de variation $(\mathrm{CV})$ a été calculé selon la formule suivante :

$\mathrm{CV}=$ (écart-type / moyenne des titres) $\mathrm{x} 100$

L'interprétation est donnée dans le tableau I.

La date de vaccination contre IBD a été calculée selon la formule de Deventer :

Age de vaccination $=\left\{\left(\log 2\right.\right.$ titre- $\left.\left.\log 2 \mathrm{~V}^{*}\right) \times \mathrm{T}\right\}+$ Age du poussin + correction $0-4$

où $\log 2 \mathrm{~V}^{*}$ était, selon le type de vaccin à utiliser, un vaccin utilisant une souche intermédiaire ; V = 125 un vaccin utilisant une souche intermédiaire plus $\mathrm{V}=500$; T la demi-vie des Ac, estimé à trois jours ; «Age du poussin », c'est-à-dire au moment de la prise du prélèvement ; « correction $0-4$ », le nombre de jours en plus si prélèvement entre 0 et 4 jours d'âge (tableau II).

\section{RESULTATS}

\section{Etude technique}

L'étude a révélé qu'aucun des centres visités n'appliquait rigoureusement le programme de vaccination préconisé par la CNPA. En effet, chaque société avait adopté son propre programme de vaccination, la conséquence étant qu'il y avait pratiquement autant de programmes de vaccination que de sociétés. La variabilité constatée tenait au type de vaccin et à la méthode de vaccination. Pour cette dernière, il y a eu de la part des propriétaires une tendance à favoriser la vaccination dans l'eau de boisson. De plus, le nombre d'interventions n'était pas le même. Il s'est avéré que les rappels de vaccins contre les NDV et IBD, préconisés vers les 50-55 semaines d'âge pour les reproducteurs, n'étaient pas administrés par le tiers des sociétés (tableau III).

\section{Etude sérologique}

La totalité des centres de reproducteurs ponte ont présenté une bonne cinétique en Ac dirigés contre le NDV (figure 2). En revanche, trois centres de reproducteurs chair sur six ont présenté un titre sérologique très bas, en dessous du seuil de protection entre les $7^{\mathrm{e}}$ et $16^{\mathrm{e}}$ semaines (figure 3 ). En tenant compte des valeurs du coefficient de variation, tous les lots de reproducteurs ponte pouvaient être considérés comme homogènes au regard de leur réponse immunitaire postvaccinale contre la NDV pendant la période de production. Le taux a été acceptable chez quatre sur six élevages de reproducteurs chair.

L'évolution du titre moyen en Ac anti-IBV a généralement été mauvaise dans toutes les analyses avec des titres parfois très hauts, sans rapport avec la vaccination, et parfois de mauvaises réponses aux sollicitations immunologiques. Chez les reproducteurs chair, les titres en Ac anti-IBV se sont stabilisés à partir de la $30^{\mathrm{e}}$ semaine d'âge dans la majorité des bâtiments. Les variations ont été observées essentiellement au cours de la période d'élevage (figure 4). L'hétérogénéité des lots en matière de titres en Ac anti-IBV a été très fréquente dans tous les bâtiments, indépendamment de l'âge des oiseaux.

Concernant la maladie de Gumboro, 50 p. 100 des bâtiments de reproducteurs (chair et ponte) avaient une cinétique conférant un seuil de protection élevé (tableau IV). Un seul bâtiment de reproducteurs chair a présenté des titres en Ac anti-IBD pratiquement nuls entre la $7^{\mathrm{e}}$ et la $16^{\mathrm{e}}$ semaine, alors que la vaccination était sensée avoir été réalisée. Le CV a été bon chez les reproducteurs ponte, tandis que la moitié des reproducteurs chair ont présenté un CV acceptable.

Si globalement les résultats ont été assez satisfaisants, chaque bâtiment pris individuellement a montré des insuffisances spécifiques au niveau de la prophylaxie appliquée. La figure 5 montre

\section{Tableau I}

Interprétation des coefficients de variation (CV)

$\begin{array}{ll}\text { CV }(\%) & \text { Interprétation } \\ <30 & \text { Lot homogène (excellent) } \\ 30 \text { à } 50 & \text { Lot intermédiaire (bon) } \\ >50 & \text { Lot hétérogène (mauvais) }\end{array}$

\section{Tableau II}

Attribution d'un facteur de correction en fonction de l'âge du poussin lors du prélèvement

\begin{tabular}{cc|}
$\begin{array}{c}\text { Age au prélèvement } \\
\text { (jour) }\end{array}$ & Correction \\
\hline 0 & 4 \\
1 & 3 \\
2 & 2 \\
3 & 1 \\
$\geq 4$ & 0 \\
\hline
\end{tabular}


Tableau III

Programme de vaccination des poules reproductrices recommandé par la Commission nationale de pathologie aviaire (2009)

\begin{tabular}{|c|c|c|c|c|c|}
\hline $\begin{array}{l}\text { Age } \\
\text { (sem.) }\end{array}$ & $\begin{array}{l}\text { Age } \\
\text { (jours) }\end{array}$ & Type de vaccin & Indication & Administration & Laboratoire / vaccin \\
\hline \multirow[t]{4}{*}{$1-2$} & Couvoir & $\begin{array}{l}\text { Marek / vivant congelé / } \\
\text { bivalent ou Marek-Gumboro / } \\
\text { immuncomplexe / recombinant }\end{array}$ & $\begin{array}{l}\text { Maladie de Marek et } \\
\text { maladie de Gumboro }\end{array}$ & $\begin{array}{l}\text { Sous-cutanée ou } \\
\text { intramusculaire }\end{array}$ & $\begin{array}{l}\text { Intervet / Nobilis Rismavac + CA } \\
126 \text { et Ceva / Cevac Transmune } \\
\text { IBD ou Merial / Vaxxitek }\end{array}$ \\
\hline & $1-10$ & Coccidiose / vivant & Coccidiose & Eau de boisson & $\begin{array}{l}\text { Biopharm / Livacox ou Shering / } \\
\text { Paracox } 8\end{array}$ \\
\hline & \multirow[t]{2}{*}{$1-5$} & $\begin{array}{l}\text { Newcastle - bronchite (HB1- } \\
\text { H120) / vivant / bivalent }\end{array}$ & $\begin{array}{l}\text { Maladie de Newcastle } \\
\text { et bronchite } \\
\text { infectieuse }\end{array}$ & $\begin{array}{l}\text { Nébulisation, } \\
\text { instillation oculaire } \\
\text { ou trempage du bec }\end{array}$ & Hipra / Hipraviar B1 / H120 \\
\hline & & $\begin{array}{l}\text { Bronchite }+ \text { Newcastle } \diamond \text { (HB1 } \\
\text { et H120) / vivants / monovalents }\end{array}$ & & & $\begin{array}{l}\text { Hipra / Bronipra-1 ou Nobilis IB } \\
\text { MA5 et Ceva / Cevac Vitapest } \\
\text { ou Ceva / Cevac Uni L }\end{array}$ \\
\hline \multirow[t]{4}{*}{$\bullet 2-3$} & $10-14$ & $\begin{array}{l}\text { Gumboro / vivant / monovalent } \\
\text { intermédiaire ou intermédiaire } \\
\text { plus }\end{array}$ & Maladie de Gumboro & Eau de boisson & $\begin{array}{l}\text { Ceva / Cevac Gumbo L ou } \\
\text { Merial / Gallivac IBD H2512 }\end{array}$ \\
\hline & $17-19 *$ & $\begin{array}{l}\text { Newcastle / vivant / monovalent } \\
\text { cloné ou LaSota ou apathogène }\end{array}$ & Maladie de Newcastle & $\begin{array}{l}\text { Nébulisation ou eau } \\
\text { de boisson }\end{array}$ & $\begin{array}{l}\text { Hipra / Hipraviar Clon ou Ceva } \\
\text { / Cevac New L ou Ceva / Cevac } \\
\text { Vitapest }\end{array}$ \\
\hline & $\bullet 19-21$ & - Réovirose / vivant S1133 & Réovirose & Sous-cutanée & Intervet / Nobilis REO 1133 \\
\hline & $21-23$ & $\begin{array}{l}\text { Gumboro / vivant / monovalent } \\
\text { intermédiaire ou intermédiaire } \\
\text { plus }\end{array}$ & Maladie de Gumboro & Eau de boisson & $\begin{array}{l}\text { Ceva / Cevac Gumbo L ou } \\
\text { Merial / Gallivac IBD H2512 }\end{array}$ \\
\hline$* 4$ & $26-28 *$ & $\begin{array}{l}\text { Bronchite / vivant / monovalent } \\
\text { H120 ou MA5 }\end{array}$ & Bronchite infectieuse & $\begin{array}{l}\text { Nébulisation ou eau } \\
\text { de boisson }\end{array}$ & $\begin{array}{l}\text { Hipra / Bronipra-1 ou Intervet / } \\
\text { Nobilis IB MA5 }\end{array}$ \\
\hline \multirow[t]{2}{*}{ • 5-6 } & \multirow[t]{2}{*}{$\bullet 35-40$} & $\begin{array}{l}\text { Newcastle / vivant / monovalent } \\
\text { cloné ou LaSota ou apathogène }\end{array}$ & Maladie de Newcastle & $\begin{array}{l}\text { Nébulisation ou eau } \\
\text { de boisson }\end{array}$ & $\begin{array}{l}\text { Hipra / Hipraviar Clon ou Ceva } \\
\text { / Cevac New L ou Ceva / Cevac } \\
\text { Vitapest }\end{array}$ \\
\hline & & - Réovirose / vivant S1133 & Réovirose & Sous-cutanée & Intervet / Nobilis REO 1133 \\
\hline 7 & 49 & Variole / vivant & Variole aviaire & Transfixion alaire & Ceva / Cevac FPL \\
\hline 10 & 70 & Encéphalomyélite / vivant & Encéphalomyélite & Eau de boisson & Merial / Myelovax \\
\hline * 11 & 宷 77 & $\begin{array}{l}\text { RTI-SIGT / vivant / } \\
\text { monovalent }\end{array}$ & Grosse-tête & $\begin{array}{l}\text { Nébulisation ou eau } \\
\text { de boisson }\end{array}$ & Hipra / Hipraviar SHS \\
\hline \multirow[t]{2}{*}{$12-14$} & 84 & $\begin{array}{l}\text { Newcastle / vivant / monovalent } \\
\text { cloné ou LaSota ou apathogène }\end{array}$ & Maladie de Newcastle & $\begin{array}{l}\text { Nébulisation ou eau } \\
\text { de boisson }\end{array}$ & $\begin{array}{l}\text { Ceva / Cevac New L ou Hipra / } \\
\text { Hipraviar Clon ou Ceva / Cevac } \\
\text { Vitapest }\end{array}$ \\
\hline & 87 & $\begin{array}{l}\text { Bronchite / vivant / monovalent } \\
\text { H120 ou MA5 }\end{array}$ & Bronchite infectieuse & $\begin{array}{l}\text { Nébulisation ou eau } \\
\text { de boisson }\end{array}$ & $\begin{array}{l}\text { Hipra / Bronipra I ou Intervet / } \\
\text { Nobilis IB MA5 }\end{array}$ \\
\hline \multirow[t]{2}{*}{ 16-18 } & \multirow[t]{2}{*}{$\begin{array}{l}\text { \$* } \\
112- \\
126\end{array}$} & $\begin{array}{l}\text { * Newcastle - bronchite - } \\
\text { grosse-tête / inactivé / } \\
\text { trivalent Gumboro / inactivé } \\
\text { / monovalent ou Newcastle - } \\
\text { bronchite - Gumboro }\end{array}$ & $\begin{array}{l}\text { Newcastle - bronchite - } \\
\text { Gumboro - grosse-tête } \\
\text { Newcastle - bronchite - } \\
\text { Gumboro }\end{array}$ & $\begin{array}{l}\text { Sous-cutanée ou } \\
\text { intramusculaire }\end{array}$ & $\begin{array}{l}\text { Hipra / Hipraviar Trt ND IB et } \\
\text { Merial / Gumboriffa ou Hipra / } \\
\text { Bronipra ND IBD }\end{array}$ \\
\hline & & • Réovirose / inactivé & Réovirose & $\begin{array}{l}\text { Sous-cutanée ou } \\
\text { intramusculaire }\end{array}$ & Ceva / Reomune III \\
\hline $35-40$ & $\begin{array}{l}245- \\
280\end{array}$ & $\begin{array}{l}\text { Newcastle - bronchite / inactivé } \\
\text { / bivalent }\end{array}$ & $\begin{array}{l}\text { Maladie de Newcastle } \\
\text { et bronchite } \\
\text { infectieuse }\end{array}$ & $\begin{array}{l}\text { Sous-cutanée ou } \\
\text { intramusculaire }\end{array}$ & Ceva / Cevac ND IB K \\
\hline $50-55$ & $\begin{array}{l}350- \\
385\end{array}$ & $\begin{array}{l}\text { Newcastle - Gumboro / inactivé } \\
\text { / bivalent ou monovalent }\end{array}$ & $\begin{array}{l}\text { Maladie de Newcastle } \\
\text { et maladie de } \\
\text { Gumboro }\end{array}$ & $\begin{array}{l}\text { Sous-cutanée ou } \\
\text { intramusculaire }\end{array}$ & $\begin{array}{l}\text { Ceva / Cevac ND G K ou } \\
\text { Ceva / Cevac ND K et Merial / } \\
\text { Gumboriffa }\end{array}$ \\
\hline
\end{tabular}

$\checkmark$ Administré séparément à deux jours d'intervalle minimum

- Vaccination contre la réovirose dans les élevages de reproductrices chair à problèmes

* Date de vaccination de la maladie de Newcastle et de la bronchite infectieuse pouvant être intervertie selon contexte régional

* Vaccination contre la grosse-tête (SIGT) dans les élevages à problèmes 


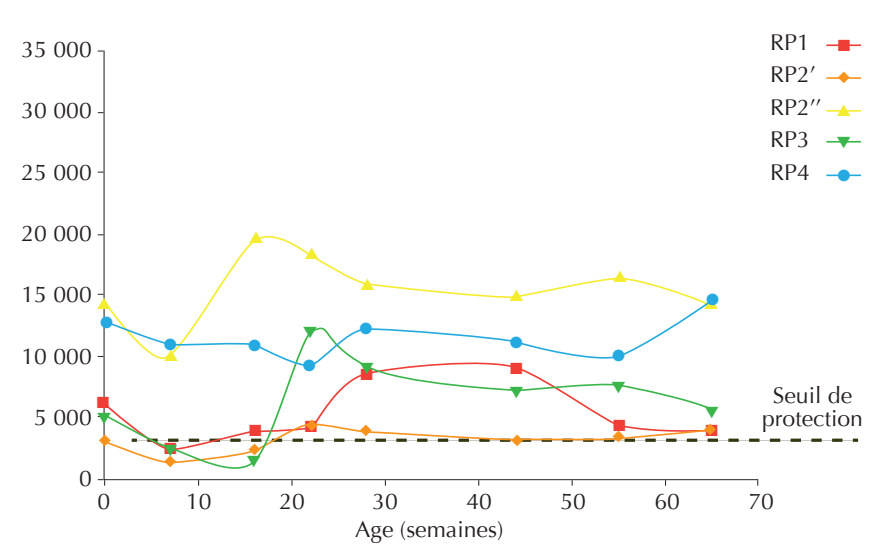

Figure 2 : cinétique hebdomadaire du titre moyen en anticorps anti-virus de la maladie de Newcastle dans les bâtiments de reproducteurs ponte $(R P)$.

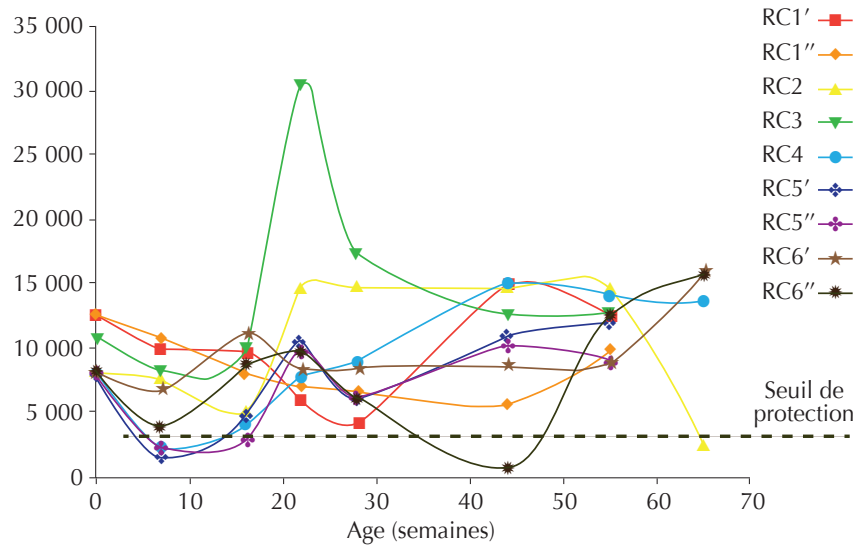

Figure 3 : cinétique hebdomadaire du titre moyen des anticorps anti-virus de la maladie de Newcastle dans les bâtiments de reproducteurs chair $(R C)$.

\section{Tableau IV}

Résultats sérologiques de la maladie de Gumboro

\begin{tabular}{|c|c|c|c|c|c|c|c|c|c|}
\hline & Semaine & $\mathbf{0}$ & 7 & 16 & 22 & 28 & 43 & 55 & 66 \\
\hline \multicolumn{10}{|c|}{ Reproducteur chair } \\
\hline PC1 Bât. 1 & $\begin{array}{c}\text { Moy. titre } \\
\text { CV }\end{array}$ & $\begin{array}{r}8629,9 \\
30,0\end{array}$ & $\begin{array}{r}7236,2 \\
18,2\end{array}$ & $\begin{array}{r}10607,3 \\
22,1\end{array}$ & $\begin{array}{r}12390,5 \\
33,7\end{array}$ & & $\begin{array}{r}10323,2 \\
35,9\end{array}$ & $\begin{array}{r}8274,8 \\
36,7\end{array}$ & $\begin{array}{r}11447,6 \\
23,9\end{array}$ \\
\hline PC1 Bât. 2 & $\begin{array}{l}\text { Moy. titre } \\
\text { CV }\end{array}$ & $\begin{array}{r}8629,9 \\
30,0\end{array}$ & $\begin{array}{r}9625,6 \\
30,3\end{array}$ & $\begin{array}{r}8827,5 \\
32,2\end{array}$ & $\begin{array}{r}13516,5 \\
16,3\end{array}$ & & $\begin{array}{r}10051,6 \\
46,9\end{array}$ & $\begin{array}{r}10544,3 \\
29,4\end{array}$ & $\begin{array}{r}11768,3 \\
30,9\end{array}$ \\
\hline PC2 Bât. 1 & $\begin{array}{c}\text { Moy. titre } \\
\text { CV }\end{array}$ & $\begin{array}{r}7535,3 \\
29,2\end{array}$ & $\begin{array}{r}4822,4 \\
31,0\end{array}$ & $\begin{array}{r}4238,0 \\
40,8\end{array}$ & $\begin{array}{r}8559,5 \\
45,9\end{array}$ & & $\begin{array}{r}8199,1 \\
26,2\end{array}$ & $\begin{array}{r}6619,7 \\
31,1\end{array}$ & $\begin{array}{r}3012,6 \\
114,2\end{array}$ \\
\hline PC2 Bât. 2 & $\begin{array}{c}\text { Moy. titre } \\
\text { CV }\end{array}$ & $\begin{array}{r}7535,3 \\
29,2\end{array}$ & $\begin{array}{r}4072,2 \\
32,5\end{array}$ & $\begin{array}{r}4605,8 \\
59,6\end{array}$ & $\begin{array}{r}10913,3 \\
17,0\end{array}$ & & $\begin{array}{r}7360,7 \\
28,8\end{array}$ & $\begin{array}{r}7115,6 \\
26,1\end{array}$ & $\begin{array}{r}6777,8 \\
66,9\end{array}$ \\
\hline PC3 & $\begin{array}{c}\text { Moy. titre } \\
\text { CV }\end{array}$ & $\begin{array}{r}8316,5 \\
35,5\end{array}$ & $\begin{array}{r}8217,9 \\
27,7\end{array}$ & $\begin{array}{r}3466,9 \\
77,6\end{array}$ & $\begin{array}{r}8483,0 \\
32,4\end{array}$ & $\begin{array}{r}2477,8 \\
100,7\end{array}$ & $\begin{array}{r}1620,4 \\
68,0\end{array}$ & $\begin{array}{r}2184,1 \\
93,2\end{array}$ & $\begin{array}{r}1685,8 \\
89,3\end{array}$ \\
\hline PC4 & $\begin{array}{c}\text { Moy. titre } \\
\text { CV }\end{array}$ & $\begin{array}{r}3674,2 \\
109,9\end{array}$ & $\begin{array}{r}2260,4 \\
89,7\end{array}$ & $\begin{array}{r}6609,4 \\
40,9\end{array}$ & $\begin{array}{r}6323,1 \\
46,9\end{array}$ & & $\begin{array}{r}7090,4 \\
48,3\end{array}$ & $\begin{array}{r}4354,4 \\
53,5\end{array}$ & $\begin{array}{r}5195,3 \\
41,2\end{array}$ \\
\hline PC5 & $\begin{array}{c}\text { Moy. titre } \\
\text { CV }\end{array}$ & $\begin{array}{r}8704,1 \\
29,0\end{array}$ & $\begin{array}{r}1896,8 \\
126,7\end{array}$ & $\begin{array}{r}8723,4 \\
17,4\end{array}$ & $\begin{array}{r}10262,5 \\
43,3\end{array}$ & & $\begin{array}{r}11601,9 \\
35,8\end{array}$ & & $\begin{array}{r}9409,2 \\
29,7\end{array}$ \\
\hline PC6 Bât. 1 & $\begin{array}{c}\text { Moy. titre } \\
\text { CV }\end{array}$ & $\begin{array}{r}15495,5 \\
38,6\end{array}$ & $\begin{array}{r}8414,4 \\
50,4\end{array}$ & $\begin{array}{r}3821,6 \\
69,1\end{array}$ & $\begin{array}{r}3313,6 \\
61,8\end{array}$ & & $\begin{array}{r}3714,7 \\
97,1\end{array}$ & $\begin{array}{r}3085,5 \\
68,3\end{array}$ & \\
\hline PC6 Bât. 2 & $\begin{array}{c}\text { Moy. titre } \\
\text { CV }\end{array}$ & $\begin{array}{r}15495,5 \\
38,6\end{array}$ & $\begin{array}{r}6099,9 \\
50,4\end{array}$ & $\begin{array}{r}3559,8 \\
69,9\end{array}$ & $\begin{array}{r}4076,8 \\
76,5\end{array}$ & & $\begin{array}{r}177,8 \\
92,0\end{array}$ & $\begin{array}{r}1446,6 \\
94,9\end{array}$ & \\
\hline \multicolumn{10}{|c|}{ Reproducteur ponte } \\
\hline PP1 Bât. 1 & $\begin{array}{c}\text { Moy. titre } \\
\text { CV\% }\end{array}$ & $\begin{array}{r}6708,7 \\
23,3\end{array}$ & $\begin{array}{r}5382,3 \\
28,4\end{array}$ & $\begin{array}{r}3998,5 \\
47,9\end{array}$ & $\begin{array}{r}5260,6 \\
44,0\end{array}$ & & $\begin{array}{r}3720,1 \\
46,6\end{array}$ & $\begin{array}{r}5482,6 \\
45,0\end{array}$ & $\begin{array}{r}5689,9 \\
28,2\end{array}$ \\
\hline PP1 Bât. 2 & $\begin{array}{c}\text { Moy. titre } \\
\text { CV\% }\end{array}$ & $\begin{array}{r}6708,7 \\
23,3\end{array}$ & $\begin{array}{r}5382,3 \\
28,4\end{array}$ & $\begin{array}{r}3998,5 \\
47,9\end{array}$ & $\begin{array}{r}5260,6 \\
44,0\end{array}$ & & $\begin{array}{r}3720,1 \\
46,6\end{array}$ & $\begin{array}{r}5482,6 \\
45,0\end{array}$ & $\begin{array}{r}5689,9 \\
28,2\end{array}$ \\
\hline PP2 & $\begin{array}{c}\text { Moy. titre } \\
\text { CV\% }\end{array}$ & $\begin{array}{r}21729,8 \\
15,6\end{array}$ & $\begin{array}{r}15576,3 \\
55,0\end{array}$ & $\begin{array}{r}19785,5 \\
30,8\end{array}$ & $\begin{array}{r}19178,7 \\
36,5\end{array}$ & & $\begin{array}{r}9336,2 \\
50,7\end{array}$ & $\begin{array}{r}11140,3 \\
25,7\end{array}$ & $\begin{array}{r}10000,9 \\
34,8\end{array}$ \\
\hline PP3 & $\begin{array}{c}\text { Moy. titre } \\
\text { CV }\end{array}$ & $\begin{array}{r}3451,1 \\
44,7\end{array}$ & $\begin{array}{r}3484,5 \\
31,3\end{array}$ & $\begin{array}{r}422,1 \\
29,0\end{array}$ & $\begin{array}{r}3010,4 \\
48,7\end{array}$ & & $\begin{array}{r}1529,9 \\
56,9\end{array}$ & $\begin{array}{r}2096,5 \\
53,8\end{array}$ & $\begin{array}{r}1807,9 \\
80,4\end{array}$ \\
\hline PP4 & $\begin{array}{c}\text { Moy. titre } \\
\text { CV }\end{array}$ & $\begin{array}{r}10255,1 \\
20,7\end{array}$ & $\begin{array}{r}8376,5 \\
18,6\end{array}$ & $\begin{array}{r}8485,7 \\
33,8\end{array}$ & $\begin{array}{r}10932,4 \\
17,6\end{array}$ & & $\begin{array}{r}14043,6 \\
27,8\end{array}$ & & $\begin{array}{r}12787,3 \\
27,6\end{array}$ \\
\hline
\end{tabular}

PC : poulet de chair ; PP : poule pondeuse

Bât. : bâtiment ; Moy. : moyenne

$\mathrm{CV}$ : coefficient de variation 
l'exemple d'un bâtiment où les auteurs ont enregistré une cinétique en Ac anti-IBD sans rapport avec les vaccinations réalisées et un pourcentage de $\mathrm{CV}$ très élevé.

De nombreuses formules permettent d'évaluer la date approximative de la primovaccination en tenant compte du titre Elisa en Ac maternels. Les résultats ont montré que la majorité des établissements vaccinaient plus tôt que la normale avec les vaccins « souches intermédiaires plus ». En outre, il y avait en général une, voire deux vaccinations de trop. Les dates d'intervention ont tenu compte du titre en Ac maternels selon la formule retenue de Deventer, car celle de Kouwenhoven exige certaines précisions vis-à-vis du type du vaccin qui n'étaient pas toujours disponibles.

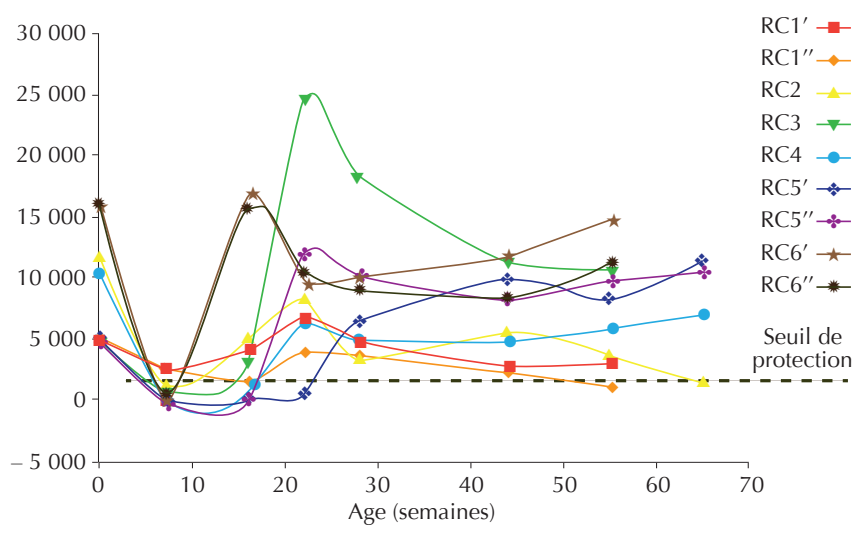

Figure 4 : cinétique hebdomadaire du titre moyen en anticorps anti-virus de la bronchite infectieuse dans les bâtiments de reproducteurs chair $(R C)$.

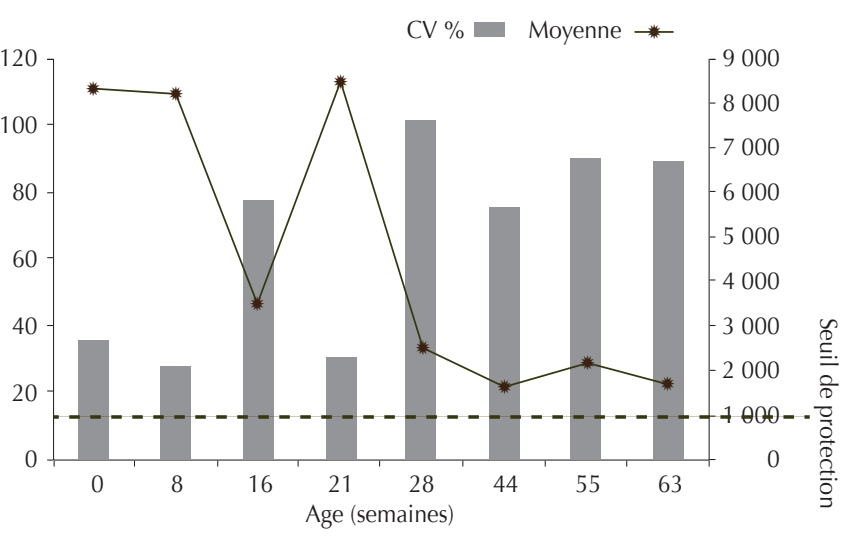

Figure 5 : cinétique des anticorps anti-virus de la maladie de Gumboro (titre moyen) et coefficient de variation (\%) dans un bâtiment de reproducteur chair (RC5).

\section{- DISCUSSION}

Les résultats du contrôle de la vaccination contre les maladies étudiées ont montré que la quasi-totalité des poussins bénéficiaient d'un stock d'Ac maternels satisfaisant et en corrélation positive avec celui des mères. Ce taux était d'autant plus important et homogène que la vaccination des poules avait été bien faite. Bien que cette protection passive ait été d'un assez haut niveau à l'éclosion, elle risquait de s'épuiser avec le temps, sachant qu'à un certain âge, le poussin se révèle sensible à l'infection (seuil de protection) (9).
Concernant les résultats sérologiques relatifs à la bronchite infectieuse, les lots étaient très hétérogènes le premier jour. Le CV s'est révélé en général assez élevé, à l'exception d'un seul bâtiment, d'une hétérogénéité assez importante et habituelle selon Alvarado et coll. (2). De même pour la maladie de Gumboro, le CV a été acceptable dans la majorité des élevages des reproducteurs chair.

La cinétique en Ac anti-IBV a été généralement mauvaise dans toutes les analyses. En effet, les titres ont parfois été très élevés quel qu'ait été l'âge des oiseaux et sans qu'il y ait eu un rapport avec la vaccination. Ceci pouvait être lié à l'existence de variants préexistants mais non pathogènes (3) ou à l'émergence de variants du virus IBV (6). Dans ce même contexte, certains auteurs confirment certaines réactions croisées en utilisant des vaccins vivants et inactivés, suivis d'épreuves virulentes par des souches homologues et/ou hétérologues du virus de la bronchite infectieuse $(4,7)$.

L'évolution des titres en Ac sériques anti-IBDV a été dans l'ensemble satisfaisante dans les élevages visités. La multiplicité des administrations de vaccins vivants pendant les trois premières semaines de vie, sans une augmentation réelle des titres en Ac, pourrait s'expliquer par une neutralisation des vaccins par les Ac maternels $(5,10)$. Cette constatation a été signalée par d'autres auteurs qui ont rapporté que le vaccin lui-même ne semblait pas toujours accélérer la chute des titres en Ac maternels (7). Dans le présent travail, les titres en Ac se sont stabilisés à partir de la $30^{\mathrm{e}}$ semaine, après l'immunisation des animaux à l'aide d'un vaccin inactivé, comme rapporté par Herdt et coll. (3).

Calculer la date précise de la primovaccination pour la maladie de Gumboro permet aux poussins de bénéficier de la prise vaccinale en économisant une, voire deux administrations de vaccins ; l'étude a révélé que la majorité des sociétés ne prenaient pas en considération ce point important et vaccinaient tôt par rapport à la date optimale. Un autre problème était celui du non-respect du nombre de vaccins contre la maladie de Gumboro dans le jeune âge. Même si les volailles ne sont plus sensibles à l'infection pendant la période de ponte, le recours aux vaccins inactivés s'impose afin de garantir une immunité satisfaisante à la descendance (8).

L'administration du vaccin dans l'eau de boisson est le moyen de vaccination le plus utilisé dans les élevages avicoles tunisiens compte tenu de la facilité de sa mise en œuvre et de la rapidité de sa réalisation. Cette méthode présente aussi l'avantage d'être peu stressante pour les volailles et peu coûteuse (main d'œuvre). En revanche, ce mode de vaccination demeure le moins efficace car la réponse immunitaire engendrée est irrégulière et le plus souvent faible (1) en raison de la mauvaise qualité chimique et/ou microbiologique de l'eau de boisson utilisée. Ainsi, la présence de métaux lourds (fer, cuivre...) inactive le virus vaccinal. Malgré cela, la vaccination dans l'eau de boisson reste la technique recommandée pour l'administration des vaccins vivants contre la maladie de Gumboro. Enfin, le choix de cette méthode est un facteur qui affecte la prise vaccinale, notamment pour la bronchite infectieuse et la maladie de Newcastle dont la vaccination nécessite l'emploi de la nébulisation pour une meilleure diffusion des virus vaccinaux concernés.

\section{CONCLUSION}

Ce travail a permis de rendre compte que, malgré le non-respect des modalités de la vaccination selon les recommandations de la CNPA, dans l'ensemble, la protection contre les principales infections virales dans les élevages reproducteurs tunisiens était satisfaisante car le poussin était de bonne qualité, et la vaccination contre la maladie de Newcastle, la bronchite infectieuse et la mala- 
die de Gumboro était correctement réalisée pendant les premières semaines. Toutefois, le nombre d'interventions était parfois exagéré faisant des charges vaccinales un handicap lourd pour la gestion d'un bâtiment de volailles reproductrices.

Pour chaque société, il faudrait établir des bases de données en vue d'une meilleure maîtrise des coûts directs et de la santé, tout en sachant que le contrôle des maladies infectieuses d'origine virale et le suivi de la prise vaccinale ne pourraient se faire sans le recours au laboratoire.

\section{BIBLIOGRAPHIE}

1. ALLAN W.H., LANCASTER J.E., TOCH B., 1978. Newcastle disease vaccine: their production and use. Rome, Italy, FAO, p. 6-19.

2. ALVARADO I.R., VILLEGAS P., EL-ATTRACHE J., BROWN T.P., 2003. Evaluation of the protection conferred by commercial vaccines against the California 99 isolate of infectious bronchitis virus. Avian Dis., 47: 1298-1304.

3. HERDT P.D., DUCATELLE R., UYTTEBROEK E., SNEEP A., TORBEYNS R., 2001. Infectious bronchitis serology in broilers and broiler breeders: Correlations between antibody titers and performance in vaccinated flocks. Avian Dis., 45: 612-619.

\section{Summary}

Cherif A., Bouslama A., Chakroun C., Turki I., Kaboudi K., Bouzouaia $\mathbf{M}$. Serological monitoring of vaccination against the major avian viral diseases in poultry breeding farms in Tunisia

A sero-epidemiological study was conducted in six broiler breeding companies and four broiler breeding laying hen companies, covering most of the poultry breeding farms in Tunisia. The objective was to evaluate the effectiveness of the immune response following the application of vaccination protocols established by the owners and carried out against the viruses of Newcastle disease (NDV), infectious bronchitis (IBV) and infectious bursal disease (IBD). During eight visits per farm, questionnaires were completed and 2000 samples were taken. The samples were submitted to indirect ELISA using CIVTEST AVINDV, -IBV, and -IBD kits. For each date and each disease, the mean specific antibody titers and the coefficient of variation were calculated. These indicators revealed that the chicks were in good health, the kinetics of post-vaccination antibodies generally satisfactory, and the overall immune response sufficiently uniform. However, the vaccination protocol recommended by the National Commission for Avian Diseases was not always applied. This either caused a temporary drop in antibody levels resulting in a high risk of infection, or necessitated interventions resulting in economic losses because of vaccine-related costs. This work shows the need for farmers to have a database for the establishment of an immunoprophylaxis program adapted to the epidemiological conditions of these farms.

Keywords: Poultry - Broiler chicken - Breeding stock - Newcastle disease - Gumboro disease - Avian infectious bronchitis Vaccination - ELISA - Tunisia.
4. KING J., 1986. Serological profiles of commercial broiler breeders and their progeny. 1. Infectious bronchitis virus. Avian Dis., 30: 719-723.

5. KNOBLICH H.V., SOMMER S.E., JACKWOOD D.J., 2000. Antibody titers to infectious bursal disease virus in broiler chicks after vaccination at one day of age with infectious bursal disease virus and Marek's disease virus. Avian Dis., 44: 874-884.

6. LADMAN B.S., POPE C.R., ZIEGLER A.F., SWIECZKOWSKI T. CALLAHAN J.M., DAVISON S., GELB J., 2003. Protection of chickens after live and inactivated virus vaccination against challenge with nephropathogenic infectious bronchitis virus PA/Wolgemuth/98. Avian Dis., 46: 938-944.

7. LIN K.Y., WANG H.C., WANG C.H., 2005. Protective effect of vaccination in chicks with local infectious bronchitis virus against field virus challenge. J. Microbiol. Immunol. Infect., 38: 25-30.

8. NAQI S.A., MARQUEZ B., SAHIN N., 1983. Maternal antibody and its effect on infectious bursal disease immunization. Avian Dis., 27: 623-631.

9. PICAUlt J.P., BENNEJEAN G., 1975. Prévention de la maladie de Newcastle : utilisation de vaccins à virus vivant et inactivé chez les poussins d'un jour porteurs d'anticorps maternels. Bull. mens. Soc. Vét. prat., 15 : 103-109.

10. SOLANO W., GIAMBRONE J.J., WILLIAMS J.C., LAUERMAN L.H., PANANGALA V.S., GARCES C., 1986. Effect of maternal antibody on timing of initial vaccination of young white Leghorn chickens against infectious bursal disease virus. Avian Dis., 30: 648-652.

Accepté le 06.04.2011

\section{Resumen}

Cherif A., Bouslama A., Chakroun C., Turki I., Kaboudi K., Bouzouaia M. Seguimiento serológico de la vacunación contra las principales virosis aviares en los criaderos de reproductores de Túnez

Se llevó a cabo un estudio sero-epidemiólogico en seis sociedades de cría de reproductores de carne y cuatro de ponedoras, cubriendo la mayor parte de las crías avícolas reproductoras en Túnez. El objetivo fue evaluar la eficacidad de la respuesta inmunitaria luego de la aplicación de los protocoles de vacunación establecidos por los propietarios y efectuados contra la enfermedad de Newcastle (NDV), la bronquitis infecciosa (IBV) y la enfermedad de Gumboro (IBD). Durante las ocho visitas Ilevadas a cabo en cada cría, se llenaron cuestionarios y se efectuaron 2000 muestras. Estas muestras se sometieron al test de ELISA indirecto, utilizando los kits CIVTEST AVI "NDV", "IBV" y "IBD". Para cada fecha y cada enfermedad, se calcularon el título medio de anticuerpos específicos y el coeficiente de variación. Estos indicadores permitieron constatar que el pollito era de buena calidad sanitaria, la cinética de los anticuerpos post vacunares generalmente satisfactoria y la respuesta inmunitaria global suficientemente homogénea. Sin embargo, el protocolo de vacunación recomendado por la Comisión nacional de patología aviar no fue siempre respetado, lo que llevaba ya sea a una caída pasajera de los anticuerpos con, como consecuencia, un riesgo elevado de infección, o a intervenciones adicionales llevando a pérdidas económicas a largo plazo relacionadas con costos de vacunas. Este estudio muestra a los criadores la necesidad de disponer de una base de datos para la puesta en obra de un programa de profilaxis médica, específica a las condiciones epidemiológicas de estos criaderos.

Palabras clave: Ave de corral - Pollo de engorde - Animal reproductor - Enfermedad de Newcastle - Enfermedad de Gumboro - Bronquitis infecciosa aviar - Vacunació - ELISA Túnez. 